secondary gain may be some lowering in the energy cost of the walk so that the patient is less tired, and this has obvious implications for long term walking capability.

A successful outcome in medicine requires that diagnosis should precede treatment. For the diplegic patient we need to improve our diagnostic capabilities by studying the moving child. The potential for improved walking may be predicted by an evaluation in a gait laboratory when the child's physical maturation is coming to a plateau at about 7 years. Many advanced gait laboratories exist and are mostly dedicated to research. What patients need are more clinically based laboratories that can make kinematic recordings formally, the video recordings obtained being used to help clinical decision making. Instrumentation may also be used to assess the degree of handicap, and multiple tests may be applied - for example, estimates of the energy cost of walking, dynamic electromyography recordings for establishment of muscle contraction patterns, and even production of vector force diagrams around the leg joints. Such scientific analysis allows guess work and intuition to give way to understanding of particular sequences of movements. Similarly, a telemetry signal of heart rate can be used to estimate the "physiological cost" of walking when the resting rate is subtracted from the exercising rate and then divided by the speed obtained during that walk.

Logical suggestions for treatment may be made when these details are provided for the clinician. For example, a dynamic flexion deformity of the knee or hip may be preventing a correct step forward in the progression line. The patient's history (especially of mobility problems) and the known static deformities discovered at the initial physical examination may be combined with a study of the kinematic and kinetic data to suggest a logical programme of surgical or other treatment.

Such analysis of the walking pattern in the presence of the child's own physiotherapist and parent will often produce suggestions that may then be tested in the clinical gait laboratory - for example, in altering the footwear or providing an ankle foot orthosis (splint) - even if operative treatment is not indicated.

Surgical thinking and procedures have also changed. The "birthday syndrome" is the reminder of sometimes inappropriate surgery being applied to a patient in a haphazard way. ${ }^{5}$ The pioneering work of Gage and Sutherland in the United States has identified surgical treatment regimens that are understandable in the context of the pathological and clinical effects of diplegia. ${ }^{67}$

A thorough assessment of gait may show that it is being obstructed by restrictions of joint range. For example, the rectus femoris muscle often prevents knee flexion in swing phase, preventing anything but a walk that has a high energy cost and is stiff kneed and spastic. In such cases a simple transfer of the tendon of this muscle may "unlock" the knee and lead not only to a huge cosmetic gain but also to a reduction in the effort of clearing the swing leg.

Other leg deformities may be dealt with in one session. Even so, bony procedures may need to precede the soft tissue releases by a few weeks so that mobilisation may begin within a few days of surgery. The hope is that a single event in hospital will lead to a clearer path to more "normal" walking. Certainly the cosmetic result is often very pleasing. ${ }^{68}$

The most important long term benefit seems likely to be a reduction in the physiological cost of walking. Preliminary studies in the clinical gait laboratory in Oswestry have confirmed this effect after 12 months in all 12 patients who were treated. This holds out the promise of continuing walking for many children with diplegia as they mature beyond childhood and adolescence.

Eliminating the spastic walk is impossible because the primary lesions cannot be altered. Debate about the treatment of the secondary effects is likely to continue, but we believe that with better diagnostic methods and more appropriate surgery performed at a correct age the end results should show some improvement in the next decade.

Director,

JOHN PATRICK

Orthotic Research and Locomotor Assessment Unit,

Robert Jones and Agnes Hunt Orthopaedic Hospital,

Oswestry, Shropshire SY10 7AG

1 Little WJ. On the influence of abnormal parturition, difficult labours, premature birth, and asphyxia neonatorum, on the mental and physical condition of the child, especially in relation to deformities. Transactions of the Obstetrical Society of Londom 1862;3:293. (Reprinted in Cerebral Palsy Bulletin 1958;1:5-36.

Beals RK. Spastic paraplegia and diplegia: an evaluation of non-surgical factors influencing the prognosis for ambulation. F Bone foint Surg [Am] 1966;48:827-46.

3 Sutherland DH, Olshen R, Cooper L, Woo SK-Y. The development of mature gait. F Bone foin Surg $[\mathrm{Am}]$ 1980;62:336-53.

Bleck EE. Locomotor prognosis in cerebral palsy. Dev Med Child Neurol 1975;17:18-25.

5 Rang M, Silver R, de la Garza J. Cerebral palsy. In: Lovell WW, Winter RB, eds. Pediatri orthopedics. 2nd ed. Vol 1. Philadelphia: Lippincott, 1986:345-96.

Gage J, Fabian D, Hicks R, Tashman S. Pre and post-operative gait analysis in patients with spastic diplegia: a preliminary report. I Pediatr Orthop 1984:4:715-25.

7 Sutherland DH, Larsen LJ, Mann R. Rectus femoris release in selected patients with cerebral palsy: preliminary report. Dev Med Child Neurol 1975;17:26-34.

8 Gage JR. Gait analysis for decision making in cerebral palsy. Bull Hosp ft Dis Orthop Inst 1983;43:147-63.

\title{
Constipation in childhood
}

\section{Treatment should be given early}

Most doctors who care for children will at some stage confront one with chronic constipation. What promises in its early stage to be a condition easily remedied by increasing the fluid and fibre intake gradually develops into a nightmare for all concerned. A mixture of painful defaecation, deliberate withholding of faeces, overflow faecal incontinence, and parental anxiety - all compounded by a physiological tendency to constipation - leads all too often to an ever increasing use of laxatives and diminishing confidence in the doctor.

Theories from child psychiatrists, ${ }^{12}$ paediatric surgeons, ${ }^{3}$ and paediatricians ${ }^{+6}$ on the aetiology of chronic constipation have been as varied as the many factors operating in each individual child. Loening-Baucke recently tried to determine which features might predict a good response to treatment.
Her regimen included the essentials: effective evacuation of retained faeces, maintenance of an empty rectum with oral or rectal medication, strict attention to diet and toilet routines, and a careful explanation of the pathophysiology. As might be expected, she found that those children who had presented with frequent overflow soiling and with faecal masses palpable on abdominal examination were less likely to be free of constipation and off laxatives after one year.

What is the place of specialist investigations? Anorectal manometry has been recommended for severly constipated children to help exclude ultrashort segment Hirschsprung's disease. ${ }^{5}$ There seems to be less inhibition of the internal sphincter muscle in response to rectal distension even in 
children without Hirschsprung's disease who are constipated when compared with those without constipation. ${ }^{8}$ LoeningBaucke also noted that in children with chronic constipation higher rectal volumes were required for maximal anal inhibition and rectal sensation was less. These findings support the theory that the degree of megarectum plays a large part in the persistence of chronic constipation. What remains unanswered is whether the megarectum is present from birth or whether it develops in response to a child's deliberate withholding of faeces for whatever reason.

How does constipation start? Many children become afraid to open their bowels as a response to episodes of painful defaecation. The pain may have been associated with an anal fissure or with rectal prolapse or may have been due simply to the passage of a large hard stool. Care should be taken to avoid a false diagnosis of child sexual abuse - chronically constipated children have good physiological reasons for having anuses with an abnormal appearance - but pain from anal abuse must be considered among the causes of faecal retention. ${ }^{9}$ Another pattern seen in many children is that they begin to avoid defaecation when first coerced to sit on a pot or a lavatory. They seem to assume that it is better to give up defaecation than run the risk of soiling their newly acquired pants and earning dishonour or worse from their parents. Once defaecation becomes associated with distress it is not surprising that they find it difficult to relax their external sphincter muscles.

A tendency to constipation is common in childhood and most children will gain confidence in defaecation as they grow older. If a child has a rectum larger than normal, however, he or she may develop chronic constipation - which in time may lead to distressing overflow faecal incontinence and major psychological problems. So treatment should be given as a matter of urgency to any child with constipation. The first step is to ensure that the child has sufficient fluid and fibre in the diet. If constipation still occurs then the use of softeners such as docusate sodium, lactulose, or methylcellulose may be sufficient to help. If not, active laxatives such as senna, bisacodyl, or magnesium hydroxide should be used to avoid the vicious cycle of faecal retention and pain. If the constipation has become chronic, and especially if the child is still a baby, investigations should be done to exclude anal anomalies or Hirschsprung's disease. Relapsing or chronic constipation, which may be associated with older childhood disorders, requires a careful liaison between paediatrician, child psychiatrist or psychologist, paediatric surgeons, and the family doctor.

Honorary Consultant Paediatrician,

G S CLAYDEN

St Thomas's Hospital, London SE1 7EH

1 Anthony EJ. An experimental approach to the psychopathology of childhood: encopresis. $\mathrm{Br} \mathcal{Y} \mathrm{Med}$ Psychol 1957;30: 146-75.

2 Berg I, Jones KV. Functional faecal incontinence in children. Arch Dis Child 1964;39:465-72.

3 Nixon HH. Megacolon and megarectum. Proceedings of the Royal Society of Medicine 1961;54: $1037-40$.

4 MacCarthy D. Encopresis. Proceedings of the Royal Society of Medicine 1976;69:19-20.

5 Clayden GS, Lawson JON. Investigation and management of long-standing chronic constipation in childhood. Arch Dis Child 1976;51:918-23.

6 Taitz LS, Wales JKH, Urwin OM Molnar D. Factors associated with outcome in management of defaecation disorders. Arch Dis Child 1986;61:472-7.

Loening-Baucke $V$. Factors determining outcome in children with chronic constipation and faecal soiling. Gut 1989;30:999-1006.

Clayden GS. Is constipation a neurodevelopmental abnormality? In: Milla PJ, ed. Disorders of gastrointestinal motility in childhood. Chichester: John Wiley, 1988:111-21.

gastrointestinal motility in childhood. Chichester: John Wiley, 1988:111-21.
Clayden GS. The reflex anal dilatation sign associated with severe chronic constipation in childhood. Arch Dis Child 1989;63:832-6.

\section{Intracranial bumps in the night}

\section{"Exploding head" syndrome is usually benign}

The transition from wakefulness to sleep is often a phase of relaxation. But it may also be a time of intense introspection, awareness of isolation, and fear. Sometimes it is associated with particular sensations or movements, which typically are brief and poorly recalled.

The movements include restless legs associated with deep paraesthesia (creeping sensations), sleep paralysis (a transient state of flaccid immobility), and myoclonic jerks. Jerks on falling asleep commonly affect a limb and are isolated single involuntary movements. ${ }^{1}$ All of these conditions are benign.

The sensory events are more varied. Among the commoner feelings is a sense of falling from a height lasting a few seconds. Gowers called this nocturnal vertigo and thought that it was common in healthy people. ${ }^{2}$ He noted that it was sometimes accompanied by a vibratory sound. $\mathrm{He}$ also referred to a sudden loud noise that disturbed sleep at its onset. ${ }^{3}$ Hypnagogic hallucinations are usually visual or auditory. In isolation they have no special links with any physical disorder, but when they are associated with narcolepsy there may also be sleep paralysis and cataplexy (muscular weakness provoked by emotional factors). The narcolepsy syndrome is a rare disorder in which circadian sleep rhythm is disturbed; it is often associated with human leucocyte antigen DR2.

A recent paper by Pearce described 50 patients with "the exploding head syndrome." 4 They complained of a loud noise in the head during the stage of twilight sleep, describing it as an explosive bang, a crack of lightning, or a loud snap. The event alarmed the victims and might wake them up. The condition was commoner in women, it covered all adult age groups, and it had often been present for many years. It was rarely familial, and there was no pain. The mechanism remains obscure.

Pearce points out that this symptom is rare in patients seen in a neurological clinic, but it may be elicited by direct questioning - some patients are reluctant to admit it. Most of the people in his series had written to him after reading his initial account of the syndrome.

What should be done for a patient who presents with this condition? The first essential is an adequate history to establish the description, the timing, and the circumstances. Anxiety and stress combined with fear are important components and may aggravate the symptoms. Questions should be asked about background factors, including drugs and alcohol, and the patient should be physically examined.

When the event is divested of overtones it will usually be found to be a purely subjective sensation during twilight sleep; it may then usually be declared benign without further investigation. Reassurance is important. When the symptoms are complex and recurrent further investigation and assessment may be needed.

Consultant Neurologist,

BRYAN ASHWORTH

Western General Hospital, Edinburgh EH4 2XU

1 Symonds CP. Nocturnal myoclonus. I Neurol Neurosurg Psychiatry 1953;16:166-71. 2 Gowers WR. Diseases of the nervous system. Vol 2. 2nd ed. Philadelphia: Blakiston, 1898:792

3 Gowers WR. The border-land of epilepsy. London: Churchill, 1907:107.

4 Pearce JMS. Clinical features of the exploding head syndrome. $I$ Neurol Neurosurg Psychiatry 1989;52:907-10. 